\title{
Non-equilibrium statistical mechanics of classical nuclei interacting with the quantum electron gas
}

\author{
Yu Wang and Lev Kantorovich
}

September 15, 2021

Department of Physics, King's College London, The Strand, London, WC2R 2LS, United Kingdom

\begin{abstract}
Kinetic equations governing time evolution of positions and momenta of atoms in extended systems are derived using quantum-classical ensembles within the Non-Equilibrium Statistical Operator Method (NESOM). Ions are treated classically, while their electrons quantum mechanically; however, the statistical operator is not factorised in any way and no simplifying assumptions are made concerning the electronic subsystem. Using this method, we derive kinetic equations of motion for the classical degrees of freedom (atoms) which account fully for the interaction and energy exchange with the quantum variables (electrons). Our equations, alongside the usual "Newtonian"-like terms normally associated with the Ehrenfest dynamics, contain additional terms, proportional to the atoms velocities, which can be associated with the "electronic friction". Possible ways of calculating the friction forces which are shown to be given via complicated non-equilibrium correlation functions, are discussed. In particular, we demonstrate that the correlation functions are directly related to the thermodynamic Matsubara Green's functions, and this relationship allows for the diagrammatic methods to be used in treating electron-electron interaction perturbatively when calculating the correlation functions. This work also generalises previous attempts, mostly based on model systems, of introducing the electronic friction into Molecular Dynamics equations of atoms.
\end{abstract}

\section{Introduction}

Classical Molecular Dynamics (MD) simulations [1, 2] play an important role in modern condensed matter physics [3] giving direct access to a wide range of statistical properties of the systems under study. In ab initio MD simulations atoms which are treated classically follow in time Newtonian's equations of motion. The latter are solved numerically using atomic forces. In $a b$ initio MD simulations the forces on atoms are calculated from the first principles by considering electrons (at each atomic configuration) entirely quantum mechanically, usually within the density functional theory (DFT) [4]. This approach is also sometimes called the mean-field approximation (MFA) [5].

Probably, the simplest quantum-mechanical justification of the MFA [6] is based on a factorisation of the density operator for the whole system into a product of individual operators for the nuclei and electrons, and then using this Ansatz in the quantum Liouville equation with subsequent replacement of the quantum bracket with the classical Poisson bracket for the classical degrees of freedom. Then, a classical trajectory is introduced by adopting a special Delta-function representation for the density operator of the classical subsystem. The important message here is that the ionic coordinates and momenta in the usual MD equations appear as statistical averages calculated at every time step. Thus, usual MD equations constitute the dynamical equations of motion (EoM) for averages as proposed originally a long time ago by Ehrenfest [7. Although more sophisticated approaches have also been developed (see e.g. 6, 8, 9, 10, and references therein) in which quantum nature of slow variables (classical degrees of freedom) is taken into account to some extent, these methods are still very complicated. At the same time, classical consideration of nuclei can still be well justified for many problems [11].

In the present paper we propose a general statistical mechanical consideration of a system consisting of slow and fast degrees of freedom assuming nuclei and electrons as a particular example. We derive EoM for slow degrees of freedom (nuclei) which interact and exchange energy with the fast degrees of freedom (electrons). Contrary to conventional approaches (e.g. 9]) based on the Liouville equation which possesses the time-reversal symmetry and is thus intrinsically equilibrium [12], our method is based on entirely non-equilibrium consideration within the Nonequilibrium Statistical Operator Method (NESOM) [12. The treatment of classical and quantum degrees of freedom 
is done within the method of mixed quantum-classical ensembles (MQCE) [13, 14] in which the Liouville operator is represented as a sum of the classical Poisson bracket and a quantum-mechanical commutator acting on the statistical operator of the whole system. The latter depends on coordinates and momenta of classical degrees of freedom and, at the same time, is a super-operator acting on quantum operators due to quantum degrees of freedom. A formal derivation of this method of treating mixed quantum-classical systems based on a group-theoretical analysis was given in Refs. 15, 16, 17. Note that in this approach the statistical operator is not assumed to be in a factorised form with respect to slow and fast degrees of freedom.

Physically, one would expect that if the "fast" electrons are in instantaneous equilibrium with the "slow" nuclei, then the former would follow the dynamics of the latter and the EoM would correspond exactly to the Ehrenfest dynamics when the nuclei move along a single trajectory (which depends on the initial conditions) while the electrons are in the ground state. However, in reality the electrons are quantum particles which impose fluctuating forces on the nuclei. Also, at a given temperature $T$, the electrons are not isolated from the nuclei and the heat bath surrounding the system; they should get enough energy to occupy an ensemble of ground and excited states corrresponding to this $T$ and will require some time to equilibrate after the nuclei displaced from their current positions. This make us think that the motion of the nuclei cannot be considered as following a single trajectory; instead, one can only consider the motion of the nuclei statistically, "on average". Moreover, the EoM for the nuclei average momenta would also deviate from the Ehrenfest dynamics: firstly, the average forces acting on the nuclei are expected to contain friction-like terms reflecting the possibility of the energy exchange, and, secondly, there should be a "conservative" force acting on the nuclei due to electrons occupying an ensemble of states. In this paper we develop a general formalism that leads to this kind of description.

We show that the EoM for nuclei corresponds to the Ehrenfest dynamics with additional terms. The latter are related to rather complicated non-equilibrium correlation functions, and we provide a way of deriving these terms systematically. In the first order approximation, our additional terms are shown to be exactly proportional to atoms momenta and can thus be interpreted as friction forces. These forces have long been known in the literature as "electronic friction" (see review [6] and references therein), but they were either introduced semi-empirically [18], as Langevin forces [11] or due to energy losses in particular model systems [6, 19, 20, 21]. In this paper we give a general derivation and justification of these kind of terms. The method presented here is a generalisation of our previous treatment [13, 14] of a classical tip of Atomic Force Microscopy interacting with quantum surface vibrations.

The plan of the paper is as follows. In the next Section we shall introduce main concepts of the NESOM and MQCE to set up the necessary definitions and notations. In Section 3 our main formalism is given and the EoM for the nuclei are derived to the first order, and we explain which quantities are used for building up this approximation. We also discuss how this procedure can be extended systematically to include terms up to any order. Although we do not consider any specific model in which the non-equilibrium correlation functions could be calculated due to an enormous complexity of those, a general discussion on how this could be done, at least in principle, will also be given. In particular, their connection to the Matsubara Green's functions [22, 23] is discussed in Appendix. Finally, in Section 4, main conclusions are drawn.

\section{NESOM and MQCE}

In NESOM statistical mechanics of a system is described in general by a statistical operator $\rho(t)$ which satisfies the Liouville equation with broken time-reversal symmetry [12]:

$$
\frac{\partial \rho}{\partial t}+i \widehat{L} \rho=-\varepsilon\left(\rho-\rho_{r e l}\right)
$$

where $\widehat{L}$ is the Liouville operator and $\rho_{\text {rel }}(t)$ is the so-called relevant distribution corresponding to local equilibrium in the system. The right hand side of Eq. (1) serves to break the time-reversal symmetry inherent to the usual Liouville equation, $\frac{\partial \rho}{\partial t}+i \widehat{L} \rho=0$, in which this term is missing, and this guarantees that the retarded solution of the Liouville equation is chosen corresponding to the physically acceptable non-equilibrium behaviour of the system. The limit $\varepsilon \rightarrow 0$ (following the thermodynamic limit) is taken after the calculation of necessary averages with respect to $\rho(t)$.

The key quantity in this formalism is the relevant distribution, $\rho_{\text {rel }}(t)$, which is constructed from a set of relevant statistical variables $X_{n}$, and follows from the principle of maximum of the information entropy. The entropy is maximum subject to the so-called self-consistency conditions stating that the statistical averages of the variables $X_{n}$ calculated with the relevant distribution, $\left\langle X_{n}\right\rangle_{\text {rel }}^{t}=\operatorname{Tr}\left(\rho_{\text {rel }}(t) X_{n}\right)$, at time $t$ are always equal to the true statistical averages, $\left\langle X_{n}\right\rangle^{t}=\operatorname{Tr}\left(\rho(t) X_{n}\right)$, calculated with the true statistical operator, $\rho(t)$. This is 
achieved using Lagrange multipliers, which makes the relevant distribution to depend explicitly on the true statistical averages of the relevant variables, and thus on the true statistical operator $\rho(t)$ which is obtained by solving the modified Liouville equation (1). This makes the whole scheme highly non-linear (and thus the term "self-consistency conditions").

When considering, within the MQCE, a mixed quantum-classical system consisting of quantum and classical degrees of freedom, one has to use a generalised expression for the Liouville operator which acts on classical variable $A$ as the classical Poisson bracket

$$
\{A, H\}=\sum_{j}\left(\frac{\partial A}{\partial Q_{j}} \frac{\partial H}{\partial P_{j}}-\frac{\partial A}{\partial P_{j}} \frac{\partial H}{\partial Q_{j}}\right)
$$

where $Q=\left\{Q_{j}\right\}$ and $P=\left\{P_{j}\right\}$ are the coordinates and momenta corresponding to the classical degrees of freedom, while it is the quantum commutator, $\frac{1}{i \hbar}[Y, H]$, when it acts on a quantum operator $Y$ associated with the quantum degrees of freedom. Here $H$ is the system total Hamiltonian, depending on both types of variables at the same time. Consequently, the true and relevant statistical operators which may also depend on both classical and quantum variables, act as operators on quantum states and, at the same time, are functions of coordinate and momenta of the classical degrees of freedom, as in classical statistical mechanics. Since the quantum operators may not necessarily commute with the Hamiltonian, one has to use the symmetrised Poisson bracket when constructing the appropriate generalised Liouville operator [15, 16, 17, 8, 24], i.e. the Liouville operator in MQCE is the sum of the quantum and classical counterparts:

$$
i \hat{L} \ldots=i \hat{L}_{q} \ldots+i \hat{L}_{c} \ldots=\frac{1}{i \hbar}[\ldots, H]+\frac{1}{2}(\{\ldots, H\}-\{H, \ldots\})
$$

i.e. it is formally constructed as a sum of the quantum and symmetrised classical Poisson brackets. It is readily seen that the Liouville operator defined in this way serves as a usual commutator when acting on quantum operators and is the classical Poisson bracket (2) when acting on classical variables. If a variable contains both classical and quantum components, the generalised operator (3) is to be used.

The statistical operator in MQCE is normalised to unity in the generalised sense, $\operatorname{Tr}(\rho)=1$, via the "total trace" defined as:

$$
\operatorname{Tr}(\ldots)=\int \operatorname{tr}(\ldots) d \Gamma
$$

where the trace written with small letters corresponds to the usual quantum trace taken with respect to the quantum states associated with the quantum degrees of freedom, while integration corresponds to all coordinates and momenta of the classical phase space $\Gamma$ as in ordinary classical statistical mechanics. Correspondingly, a statistical average of an arbitrary observable $A$, which may depend on classical degrees of freedom and, at the same time, is an operator in the quantum subspace, is defined in the generalised sense as

$$
\langle A\rangle^{t}=\operatorname{Tr}(\rho(t) A)
$$

One can also define the "flux" $\dot{A}$ operator (time derivative of $A$ ) associated with the variable $A$ in the usual way as $\dot{A}=i \hat{L} A$. It is seen that all equations look identical to either pure classical and quantum cases, only the actual meaning of the Liouville operator is different.

In the following, we shall limit ourselves with the Hamiltonian of the form

$$
H=\sum_{j} \frac{P_{j}^{2}}{2 M_{j}}+U(Q)+H_{q}(p, q ; Q)
$$

which corresponds to the electron-ion system in which ions of masses $M_{j}$ are considered classically, while electrons quantum mechanically. The index $j$ corresponds to a classical degree of freedom (i.e. each atom contributes three such degrees of freedom). Above, the first term gives the kinetic energy of the classical ions, their potential energy in an external field as well as the ion-ion interaction is provided by the second term. The last term forms the quantum Hamiltonian for the electrons (with coordinates $q=\left\{\mathbf{r}_{k}\right\}$ and momenta $p$ ),

$$
H_{q}(p, q ; Q)=H_{e}(p, q)+\Phi(Q, q)
$$

This Hamiltonian describes kinetic and interaction energies of the electrons (the first term), while their interaction with the classical coordinates, $Q$, is described by the second term. Note that interaction between the two subsystems 
in $H$ depends only on their coordinates. Then the following expression for the classical part of the Liouville operator acting on the operator $A$ is obtained:

$$
i \hat{L}_{c} A=\sum_{j}\left[\frac{P_{j}}{M_{j}} \frac{\partial A}{\partial Q_{j}}+\frac{1}{2}\left(\frac{\partial A}{\partial P_{j}} \dot{P}_{j}+\dot{P}_{j} \frac{\partial A}{\partial P_{j}}\right)\right]
$$

where the variable $\dot{P}_{j}=i \hat{L} P_{j}=i \hat{L}_{c} P_{j}=-\frac{\partial H}{\partial Q_{j}}$ corresponds to the force acting on the classical coordinate $Q_{j}$ (degree of freedom $j$ ).

When deriving kinetic equations (equations of motion for the statistical averages $\langle A\rangle^{t}$ ), the following identity proves to be indispensable:

$$
\operatorname{Tr}((i \hat{L} \rho) A)=-\operatorname{Tr}((i \hat{L} A) \rho)
$$

where the generalised Liouville operator of Eq. (3) is used. Since this identity is linear with respect to the Liouville operator, it can be proven separately for each of the Poisson brackets. For the quantum bracket, $i \hat{L}_{q}$, it follows trivially from the cyclic invariance of the quantum trace [12. To prove it for the classical Poisson brackets, one writes:

$$
\operatorname{Tr}\left(\left(i \hat{L}_{c} \rho\right) A\right)=\sum_{j} \int d \Gamma\left[\frac{P_{j}}{M_{j}} \operatorname{tr}\left(\frac{\partial \rho}{\partial Q_{j}} A\right)+\frac{1}{2} \operatorname{tr}\left(\frac{\partial \rho}{\partial P_{j}} \dot{P}_{j} A\right)+\frac{1}{2} \operatorname{tr}\left(\dot{P}_{j} \frac{\partial \rho}{\partial P_{j}} A\right)\right]
$$

Using integration by parts with respect to $Q_{j}$ for the first term in the square brackets and the fact that the density operator should vanish at the boundaries of the phase space, we find that we can replace the $\operatorname{trace} \operatorname{tr}\left(\frac{\partial \rho}{\partial Q_{j}} A\right)$ with $-\operatorname{tr}\left(\frac{\partial A}{\partial Q_{j}} \rho\right)$. Similar method is applied to the other two terms: using integration by parts with respect to $P_{j}$, cyclic invariance of the quantum trace and the fact that ionic forces $\dot{P}_{j}$ do not depend on the momentum $P_{j}$, we obtain the following substitutions for the second and the third traces in the square brackets above: $\operatorname{tr}\left(\frac{\partial \rho}{\partial P_{j}} \dot{P}_{j} A\right) \rightarrow$ $-\operatorname{tr}\left(\dot{P}_{j} \frac{\partial A}{\partial P_{j}} \rho\right)$ and $\operatorname{tr}\left(\dot{P}_{j} \frac{\partial \rho}{\partial P_{j}} A\right) \rightarrow-\operatorname{tr}\left(\frac{\partial A}{\partial P_{j}} \dot{P}_{j} \rho\right)$. This proves Eq. (9) for any quantum-classical operator $A$.

Finally, we prove that for any general operator $\widehat{B}(P, Q)$, acting on quantum states and depending on classical variables as well, the following identity is satisfied:

$$
\operatorname{Tr}\left(i L_{c} \widehat{B}\right)=0
$$

provided that the operator $\widehat{B}$ vanishes at the boundaries of the classical phase space. This is proven by using an explicit expression for the classical Liouville operator, Eq. (88). Indeed, consider the first part of it, containing the product of the classical momentum $P_{j}$ and the derivative $\frac{\partial \widehat{B}}{\partial Q_{j}}$. When taking the trace, the integration over $Q_{j}$ is performed immediately resulting in the difference $\left.\widehat{B}(P, Q)\right|_{Q_{i}=\infty}-\left.\widehat{B}(P, Q)\right|_{Q_{i}=-\infty}$ which is zero due to our assumption concerning the operator $\widehat{B}$. Similarly, the other two terms of the classical Liouville operator, Eq. (8) , also result in the zero contribution due to integration over $P_{j}$ and the fact that the force, $\dot{P}_{j}$, does not depend on the momenta.

\section{Theory}

\subsection{Relevant variables and distribution}

As we are primarily interested in this work with the equation of motion (EoM) for the classical variables which are much slower than their quantum counterparts, it is reasonable to sample over the fast degrees of freedom. In practice, this is achieved by choosing classical coordinates and momenta $Q$ and $P$ as the appropriate relevant variables. Correspondingly, the relevant distribution maximising the information entropy at the given temperature $T$ and number of electrons $N$ and subject to the self-consistency conditions,

$$
\left\langle Q_{j}\right\rangle_{r e l}^{t}=\left\langle Q_{j}\right\rangle^{t},\left\langle P_{j}\right\rangle_{r e l}^{t}=\left\langle P_{j}\right\rangle^{t}
$$

is (cf. [12]):

$$
\rho_{\text {rel }}(t)=\frac{1}{Z} \exp \left\{-\beta\left[\mathcal{H}-\sum_{j}\left(V_{j} P_{j}+\mathcal{F}_{j} Q_{j}\right)\right]\right\}
$$


where $\beta=1 / k_{B} T$ is the inverse temperature, $\mathcal{H}=H-\mu N$ is the system Hamiltonian containing explicitly the chemical potential $\mu$ of electrons, $V_{j}$ and $\mathcal{F}_{j}$ are the corresponding Lagrange multipliers and $Z$ is the normalisation factor ensuring that $\operatorname{Tr}\left(\rho_{r e l}\right)=1$. Note that the sum over $N$ is incorporated into the definition of the "small trace". The relevant statistical operator depends on time only via the Lagrange multipliers (see below).

At this point it is also convenient to introduce the statistical operator,

$$
\rho_{e q}=Z_{e q}^{-1} \exp \left(-\beta \mathcal{H}_{q}\right)
$$

for the quantum subsystem, $Z_{e q}=\operatorname{tr}\left(\exp \left(-\beta \mathcal{H}_{q}\right)\right)$, where $\mathcal{H}_{q}=H_{q}-\mu N$ is the total electronic Hamiltonian (7)) It corresponds to the quantum equilibrium canonical statistical operator for the electrons when all classical variables are fixed (i.e. the classical subsystem is frozen). Then, the relevant distribution can also be written as a product

$$
\rho_{\text {rel }}=\rho_{e q} f(P, Q, t)
$$

of a "classical distribution function"

$$
f(P, Q, t)=\frac{Z_{e q}}{Z} \exp \left\{-\beta\left[\sum_{j}\left(\frac{P_{j}^{2}}{2 M_{j}}-V_{j} P_{j}-\mathcal{F}_{j} Q_{j}\right)+U(Q)\right]\right\}, \quad \int f(P, Q, t) d \Gamma=1
$$

and the canonical quantum equilibrium statistical operator, $\rho_{e q}$. We shall also need the reduced distribution function, $f(Q, t)$, which is obtained from the distribution function above after integrating over the momenta:

$$
f(Q, t)=\int f(P, Q, t) d P=\frac{Z_{e q}}{Z_{Q}} e^{-\beta\left(U-\sum_{j} \mathcal{F}_{j} Q_{j}\right)}, \int f(Q, t) d Q=1
$$

where $Z_{Q}$ is the corresponding normalisation factor. Note that $Z_{e q}=Z_{e q}(Q)$. The average with respect to the relevant distribution of any classical variable (depending only on classical degrees of freedom) is obtained as the average with respect to the distribution function $f(P, Q, t)$; if the variable depends only on the classical coordinates, then the relevant distribution average is expressed as the average with respect to the $Q$-only distribution $f(Q, t)$.

The Lagrange multipliers are obtained from the self-consistency conditions (11). Due to explicit dependence of $\rho_{\text {rel }}$ on the ions momenta via $f(P, Q, t)$, calculation of $\left\langle P_{j}\right\rangle_{\text {rel }}^{t}$ is straightforward: it gives simply $\left\langle P_{j}\right\rangle_{r e l}^{t}=M_{j} V_{j} \equiv$ $\left\langle P_{j}\right\rangle^{t}$, and we obtain:

$$
V_{j}=\frac{\left\langle P_{j}\right\rangle^{t}}{M_{j}}
$$

Thus, $V_{j}$ has the meaning of the average velocity of the degree of freedom $j$.

Integrating with respect to all classical momenta in $\left\langle Q_{j}\right\rangle_{\text {rel }}^{t}$, one finds that $\left\langle Q_{j}\right\rangle_{\text {rel }}^{t}=\left\langle Q_{j}\right\rangle^{t}$ does only depend on the Lagrange multipliers $\left\{\mathcal{F}_{j}\right\}$. Inversely, this means that the Lagrange multipliers $\mathcal{F}_{j}$ only depend on the average coordinates $\left\{\left\langle Q_{j}\right\rangle^{t}\right\}$. To obtain an explicit expression for $\mathcal{F}_{j}$, we calculate $\left\langle\dot{P}_{j}\right\rangle_{r e l}^{t}=\left\langle-\frac{\partial H}{\partial Q_{j}}\right\rangle_{r e l}^{t}$, using Eq. (12). To this end, we first take the quantum trace of the identity (see, e.g. [12])

$$
\frac{\partial}{\partial Q_{j}} e^{-\beta H}=-\beta \int_{0}^{1} e^{-x \beta H} \frac{\partial H}{\partial Q_{j}} e^{x \beta H} e^{-\beta H} d x
$$

giving

$$
-\frac{1}{\beta} \operatorname{tr}\left(\frac{\partial}{\partial Q_{j}} e^{-\beta H}\right)=\operatorname{tr}\left(\frac{\partial H}{\partial Q_{j}} e^{-\beta H}\right)
$$

Therefore, using this and the integration by parts with respect to $Q_{j}$ in the $\left\langle-\frac{\partial H}{\partial Q_{j}}\right\rangle_{r e l}^{t}$, one obtains:

$$
\left\langle\dot{P}_{j}\right\rangle_{\text {rel }}^{t}=-\mathcal{F}_{j}
$$

Thus, the second Lagrange multiplier, $\mathcal{F}_{j}$, has the meaning of the minus average force acting on the classical degree of freedom $j$. Using the explicit expression for the Hamiltonian, Eq. (6), the ionic force $\dot{P}_{j}$ can be broken down into conservative,

$$
F_{j}^{c}=-\frac{\partial U}{\partial Q_{j}}+\left\langle X_{j}\right\rangle_{e q}
$$


and the stochastic, $\Delta X_{j}=X_{j}-\left\langle X_{j}\right\rangle_{e q}$, parts, $\dot{P}_{j}=F_{j}^{c}+\Delta X_{j}$, where $X_{j}=-\frac{\partial \Phi}{\partial Q_{j}}$ is the instantaneous force acting on the degree of freedom $j$ due to the electronic (i.e. quantum) subsystem. Therefore, using Eq. (14), one obtains that

$$
\mathcal{F}_{j}=-\int f(P, Q, t)\left\langle\dot{P}_{j}\right\rangle_{e q} d \Gamma=-\int f(P, Q, t) F_{j}^{c} d \Gamma=-\left\langle F_{j}^{c}\right\rangle_{r e l}^{t}
$$

which demonstrates that the Lagrange multiplier $\mathcal{F}_{j}$ corresponds to the average of the conservative force.

By definition, $\left\langle\Delta X_{j}\right\rangle_{e q}=0$. However, using Eq. (14), one can also check that the average of the stochastic part of the force with respect to the relevant distribution is also zero:

$$
\left\langle\Delta X_{j}\right\rangle_{r e l}^{t}=0
$$

The relevant distribution has a number of properties which are proven to be useful in our forthcoming analysis. Firstly, since $\rho_{\text {rel }}$ is equal to a product of a part, depending only on classical variables, and the quantum operator, $e^{-\beta H_{q}}$, it commutes with the Hamiltonian, $H$, Eq. (6). Therefore,

$$
i \hat{L}_{q} \rho_{\text {rel }}=0 \text { and } e^{i \hat{L}_{q} t} \rho_{\text {rel }}\left(t^{\prime}\right)=\rho_{\text {rel }}\left(t^{\prime}\right)
$$

Next, consider the relevant distribution average $\left\langle\dot{P}_{j} \psi(Q)\right\rangle_{\text {rel }}^{t}$, where $\psi(Q)$ is some function of the classical coordinates. Using the explicit expression for $\rho_{r e l}$, Eq. (12), trace identity (19) and integration by parts, one obtains:

$$
\left\langle\dot{P}_{j} \psi(Q)\right\rangle_{r e l}^{t}=-\frac{1}{\beta}\left\langle\frac{\partial \psi}{\partial Q_{j}}\right\rangle_{r e l}^{t}+\left\langle F_{j}^{c}\right\rangle_{r e l}^{t}\langle\psi(Q)\rangle_{r e l}^{t}
$$

Note that $\dot{P}_{j}$ inside the angle brackets in the left hand side of this formula can be replaced with $F_{j}^{c}$ since $\dot{P}_{j}=$ $F_{j}^{c}+\Delta X_{j}$, and the quantum equilibrium average of the stochastic force is equal to zero:

$$
\left\langle\Delta X_{j} \psi\right\rangle_{r e l}^{t}=\int \psi(Q) f(P, Q, t) \operatorname{tr}\left(\rho_{e q} \Delta X_{j}\right) d \Gamma \sim\left\langle\Delta X_{j}\right\rangle_{e q}=0
$$

due to Eq. (14). Therefore, by taking $\psi=Q_{i}$ and $\psi=F_{i}^{c}$, we obtain the following two useful relationships:

$$
\begin{gathered}
\left\langle F_{j}^{c} Q_{i}\right\rangle_{r e l}^{t}-\left\langle F_{j}^{c}\right\rangle_{r e l}^{t}\left\langle Q_{i}\right\rangle^{t}=-\frac{1}{\beta} \delta_{i j} \\
\left\langle F_{j}^{c} F_{i}^{c}\right\rangle_{r e l}^{t}-\left\langle F_{j}^{c}\right\rangle_{r e l}^{t}\left\langle F_{i}^{c}\right\rangle_{r e l}^{t}=-\frac{1}{\beta}\left\langle\frac{\partial F_{i}^{c}}{\partial Q_{j}}\right\rangle_{r e l}^{t}
\end{gathered}
$$

Since the left hand side in the second identity is symmetrical with respect to indices $i$ and $j$, we also have the symmetry relation for the relevant average of the derivative of the conservative force: $\left\langle\frac{\partial F_{j}^{c}}{\partial Q_{i}}\right\rangle_{r e l}^{t}=\left\langle\frac{\partial F_{i}^{c}}{\partial Q_{j}}\right\rangle_{r e l}^{t}$.

Another useful expression is obtained by differentiating both sides of

$$
\left\langle Q_{i}\right\rangle^{t}=\left\langle Q_{i}\right\rangle_{r e l}^{t}=\int \operatorname{tr}\left(Q_{i} \rho_{r e l}\right) d \Gamma=\int Q_{i} f(P, Q, t) d \Gamma
$$

with respect to $\left\langle Q_{j}\right\rangle^{t}$. Recalling that only the Lagrange multipliers $\left\{\mathcal{F}_{j}\right\}$ in $f(P, Q, t)$ (both in the exponential and in the $Z$ ) depend explicitly on $\left\langle Q_{i}\right\rangle^{t}$, we obtain:

$$
\frac{1}{\beta} \delta_{i j}=\sum_{j} \frac{\partial \mathcal{F}_{i}}{\partial\left\langle Q_{j}\right\rangle^{t}} \mathcal{L}_{i j}
$$

where $\mathcal{L}=\left\|\left\langle Q_{i} Q_{j}\right\rangle_{\text {rel }}^{t}-\left\langle Q_{i}\right\rangle^{t}\left\langle Q_{j}\right\rangle^{t}\right\|$ is a symmetric matrix. It follows from this that the derivative $\frac{\partial \mathcal{F}_{i}}{\partial\left\langle Q_{j}\right\rangle^{t}}=\frac{\partial \mathcal{F}_{j}}{\partial\left\langle Q_{i}\right\rangle^{t}}$ is also symmetric. Because of Eq. (22), the derivative of $\left\langle F_{j}^{c}\right\rangle_{r e l}^{t}$ with respect to $\left\langle Q_{i}\right\rangle^{t}$ is also symmetric:

$$
\frac{\partial\left\langle F_{i}^{c}\right\rangle_{r e l}^{t}}{\partial\left\langle Q_{j}\right\rangle^{t}}=\frac{\partial\left\langle F_{j}^{c}\right\rangle_{r e l}^{t}}{\partial\left\langle Q_{i}\right\rangle^{t}}
$$


To obtain a perturbative solution of the Liouville equation in Section 3.3, some other expressions involving the relevant distribution are needed. By differentiating $\rho_{\text {rel }}$ of Eq. (12) with respect to the classical coordinates and momenta, one gets:

$$
\begin{gathered}
\frac{\partial \rho_{r e l}(t)}{\partial P_{j}}=\frac{\beta}{M_{j}}\left(\left\langle P_{j}\right\rangle^{t}-P_{j}\right) \rho_{r e l} \\
\frac{\partial \rho_{r e l}(t)}{\partial Q_{j}}=\beta\left[-\left\langle\dot{P}_{j}\right\rangle_{r e l}^{t}+F_{j}^{c}+\int_{0}^{\beta} \widetilde{\Delta X}_{j}(i \lambda \hbar) d \lambda\right] \rho_{r e l}
\end{gathered}
$$

where we have used Eqs. (17), (18) and (22), and $\widetilde{\Delta X}_{j}(x)=e^{i x \mathcal{H}_{q} / \hbar} \Delta X_{j} e^{-i x \mathcal{H}_{q} / \hbar}$ is the stochastic force in the Heisenberg representation.

Finally, one can also calculate derivatives of $\rho_{\text {rel }}$ with respect to the Lagrange multipliers, remembering that the "partition function" $Z$ also depends on them:

$$
\begin{aligned}
& \frac{\partial \rho_{r e l}(t)}{\partial V_{j}}=\beta\left(P_{j}-\left\langle P_{j}\right\rangle^{t}\right) \rho_{r e l}(t) \\
& \frac{\partial \rho_{r e l}(t)}{\partial \mathcal{F}_{j}}=\beta\left(Q_{j}-\left\langle Q_{j}\right\rangle^{t}\right) \rho_{r e l}(t)
\end{aligned}
$$

\subsection{Equations of motion for ions}

In order to derive EoM for ions, we should calculate the time derivatives of the exact averages $\left\langle P_{j}\right\rangle^{t}$ and $\left\langle Q_{j}\right\rangle^{t}$. Using the Liouville equation with broken time-reversal symmetry, Eq. (1), and identity (9), one obtains:

$$
\frac{d}{d t}\left\langle Q_{n}\right\rangle^{t}=\operatorname{Tr}\left(\frac{\partial \rho(t)}{\partial t} Q_{n}\right)=\operatorname{Tr}\left(\rho\left(i \hat{L} Q_{n}\right)\right)=\frac{\left\langle P_{n}\right\rangle^{t}}{M_{n}}
$$

where we have also used the fact that $i \hat{L} Q_{n}=i \hat{L}_{c} Q_{n}=P_{n} / M_{n}$. Note that the term in the right hand side of the Liouville equation (1) does not contribute due to the self-consistency conditions (11). Similarly, using Eq. (9), we get:

$$
\frac{d}{d t}\left\langle P_{n}\right\rangle^{t}=\operatorname{Tr}\left(\rho\left(i \hat{L} P_{n}\right)\right)=\left\langle\dot{P}_{n}\right\rangle^{t}=\left\langle\dot{P}_{n}\right\rangle_{r e l}^{t}+\operatorname{Tr}\left(\dot{P}_{n} \Delta \rho\right)
$$

where $\Delta \rho=\rho-\rho_{r e l}$ is the difference between the exact and the relevant statistical operators. The obtained EoMs are similar to Newtonian ones of the ordinary Molecular Dynamics [1, 2] since the right hand side of Eq. (33) corresponds to the actual force $\left\langle\dot{P}_{n}\right\rangle^{t}$ acting on degree of freedom $j$. However, this force depends, in a rather non-trivial way, on the time evolution of the exact statistical operator $\rho(t)$ which satisfies the Liouville equation (11). Note that $\rho(t)$ is the statistical operator for the whole system, comprising both nuclei and electrons, and no attempt has been made to factorise $\rho$ in any way here.

The ion force $\left\langle\dot{P}_{n}\right\rangle_{\text {rel }}^{t}$ calculated with respect to the relevant distribution depends explicitly on time via the Lagrange multipliers; the latter are some functions of the exact expectation values $\left\langle P_{j}\right\rangle^{t}$ and $\left\langle Q_{j}\right\rangle^{t}$ as it has been discussed above. Similarly, the trace in the right hand side of Eq. (33) would depend on the Lagrange multipliers and thus on the same expectation values. One can see that the kinetic equations written above correspond to some non-linear differential equations for the observables $\left\langle P_{j}\right\rangle^{t}$ and $\left\langle Q_{j}\right\rangle^{t}$. To obtain these equations in the explicit form, we have to obtain an explicit expression for $\Delta \rho(t)$ by solving the Liouville equation (11). This will be done in the next subsection using a kind of a perturbation theory (cf. Refs. [14, 13]) in which the square root of the relative mass of the electron and nucleus, $\sqrt{m / M}$, is used as a small parameter.

\subsection{Perturbative solution of the Liouville equation: the first order}

Formally, the exact solution of the Liouville equation (11) with respect to the $\Delta \rho(t)$ can be written as [12]

$$
\Delta \rho(t)=-\int_{-\infty}^{0} d s e^{\varepsilon s} e^{i s \widehat{L}}\left(\frac{\partial}{\partial r}+i \widehat{L}\right) \rho_{r e l}(r)
$$


where $r=t+s$. Here $\widehat{L}$ is the combined Liouville operator, Eq. (3), containing both classical and quantum parts. In order to apply the perturbation theory, we have to calculate the quantity $\left(\frac{\partial}{\partial r}+i \widehat{L}\right) \rho_{\text {rel }}(r)$. This calculation consists of several steps which will be outlined below.

Firstly, $i \widehat{L} \rho_{\text {rel }}=i \widehat{L}_{c} \rho_{\text {rel }}$ due to Eq. (24). The action of the classical Liouville operator (8) on the relevant distribution is obtained using Eqs. (28) and (29) and is as follows:

$$
\begin{aligned}
i \widehat{L}_{c} \rho_{r e l}(r)=\sum_{j} & \frac{\beta}{M_{j}}\left\{\left[\left\langle P_{j}\right\rangle^{r} F_{j}^{c}-P_{j}\left\langle F_{j}^{c}\right\rangle_{r e l}^{r}+P_{j} \int_{0}^{\beta} \widetilde{\Delta X}_{j}(i \lambda \hbar) d \lambda\right] \rho_{r e l}(r)\right. \\
+ & \left.\frac{1}{2}\left(\left\langle P_{j}\right\rangle^{r}-P_{j}\right)\left(\Delta X_{j} \rho_{r e l}(r)+\rho_{r e l}(r) \Delta X_{j}\right)\right\}
\end{aligned}
$$

To calculate the time derivative of the relevant distribution, we note that it comes entirely from the Lagrange multipliers. The latter depend on time through the observables $\left\langle P_{j}\right\rangle^{t}$ and $\left\langle Q_{j}\right\rangle^{t}$ which satisfy Eqs. (33) and (32), respectively. Also, we note that if $V_{j}$ depends directly only on $\left\langle P_{j}\right\rangle^{t}$, the other Lagrange multiplier, $\mathcal{F}_{j}$, depends on all average coordinates $\left\{\left\langle Q_{j}\right\rangle^{t}\right\}$. Therefore,

$$
\begin{gathered}
\frac{\partial \rho_{r e l}(r)}{\partial r}=\sum_{j}\left(\frac{\partial \rho_{r e l}(r)}{\partial V_{j}} \frac{\partial V_{j}}{\partial r}+\frac{\partial \rho_{r e l}(r)}{\partial \mathcal{F}_{j}} \frac{\partial \mathcal{F}_{j}}{\partial r}\right) \\
=\sum_{j} \frac{\beta}{M_{j}}\left(P_{j}-\left\langle P_{j}\right\rangle^{r}\right)\left[\left\langle\dot{P}_{j}\right\rangle_{r e l}^{r}+\operatorname{Tr}\left(\dot{P}_{j} \Delta \rho(r)\right)\right] \rho_{r e l}(r) \\
-\sum_{i j} \frac{\beta}{M_{i}} \frac{\partial\left\langle F_{j}^{c}\right\rangle_{r e l}^{r}}{\partial\left\langle Q_{i}\right\rangle^{r}}\left(Q_{j}-\left\langle Q_{j}\right\rangle^{r}\right)\left\langle P_{i}\right\rangle^{r} \rho_{r e l}(r)
\end{gathered}
$$

where the use have been made of Eqs. (30)-(33) as well.

Thus, the required quantity $\left(\frac{\partial}{\partial r}+i \widehat{L}\right) \rho_{\text {rel }}(r)$ is available now as a sum of two expressions, (35) and (36), given above. These are to be acted with the exponential Liouville operator, $e^{i s \widehat{L}}$ (see Eq. (34)). In turn, the Liouville operator consists of the quantum, $i \widehat{L}_{q}$, and classical, $i \widehat{L}_{c}$, parts. Similarly to the argument of Refs. [13, 14], we argue that the classical part of $i \widehat{L}$ can be considered as being much smaller than its quantum counterpart. Indeed, on average, one can assume that the classical momentum $P_{j}$ is of the order of $M^{1 / 2}$, where $M$ is a characteristic mass of the ions. Then, according to Eq. (18), $i \widehat{L}_{c} \sim M^{-1 / 2}$ since the forces $\dot{P}_{j}$ depend only on ionic positions, not on their masses. Hence, the exponential operator, $e^{i s \widehat{L}}=e^{s\left[i \widehat{L}_{q}+i \widehat{L}_{c}\right]}$, can be expanded in a power series with respect to the "small" Liouville operator $i \widehat{L}_{c}$. This can be done systematically (and expressed via the time-ordered exponential operator, see, e.g., Chapter 6.1.1 in [23]); here we shall only need the first two terms:

$$
e^{i s \widehat{L}}=\left[1+\int_{0}^{1} e^{i x s \widehat{L}_{q}}\left(i s \widehat{L}_{c}\right) e^{-i x s \widehat{L}_{q}} d x+\ldots\right] e^{i s \widehat{L}_{q}}
$$

In this Section we shall limit ourself with the very first term in this expansion, i.e. we replace the combined Liouville operator in Eq. (34) with its quantum part. Then, since the action of the quantum exponential Liouville operator on any quantum operator $A$ is simply equal to its Hermitian conjugate, $e^{i s \widehat{L}_{q}} A=\widetilde{A}(s)$, we obtain in this order of the perturbation theory, using Eqs. (35) and (36):

$$
\begin{gathered}
\Delta \rho(t)=-\int_{-\infty}^{0} d s e^{\varepsilon s}\left\{\sum _ { j } \frac { \beta } { M _ { j } } \left[\left\langle P_{j}\right\rangle^{r}\left(F_{j}^{c}-\left\langle F_{j}^{c}\right\rangle_{r e l}^{r}\right)+\left(P_{j}-\left\langle P_{j}\right\rangle^{r}\right) \operatorname{Tr}\left(\dot{P}_{j} \Delta \rho(r)\right)\right.\right. \\
\left.+P_{j} \int_{0}^{\beta} \widetilde{\Delta X}_{j}(i \lambda \hbar+s) d \lambda\right] \rho_{r e l}(r)-\sum_{j} \frac{\beta}{2 M_{j}}\left(P_{j}-\left\langle P_{j}\right\rangle^{r}\right)\left(\widetilde{\Delta X}_{j}(s) \rho_{r e l}(r)+\rho_{\text {rel }}(r) \widetilde{\Delta X}_{j}(s)\right) \\
\left.-\sum_{i j} \frac{\beta}{M_{i}}\left\langle P_{i}\right\rangle^{r} \frac{\partial\left\langle F_{j}^{c}\right\rangle_{r e l}^{r}}{\partial\left\langle Q_{i}\right\rangle^{r}}\left(Q_{j}-\left\langle Q_{j}\right\rangle^{r}\right) \rho_{r e l}(r)\right\}
\end{gathered}
$$


It is seen that this is actually an integral equation for $\Delta \rho$ since it is present inside the integral (and the trace) as well. Therefore, it can be solved by iterations. To do this, we must analyse every term in the above expression with respect to the small parameter of our perturbation theory. It follows then that each term, apart from the term with the trace, is of the order of $P / M \sim M^{-1 / 2}$ (because of Eq. (27), the derivative $\frac{\partial\left\langle F_{j}^{c}\right\rangle_{r e l}^{r}}{\partial\left\langle Q_{i}\right\rangle^{r}}$ should be considered as of the zero order with respect to $M$ ). Thus, in order to obtain $\Delta \rho(t)$ in the first order, one can simply drop the trace term in the right hand side. We then immediately see that this particular approximation for $\Delta \rho$, which will be referred to in the following as $\Delta \rho_{1}$, does not break down the normalisation of the density operator since, as it can easily be checked by direct calculation, $\operatorname{Tr}\left(\Delta \rho_{1}\right)=0$.

Hence, dropping the trace term above, substituting the resulting expression for $\Delta \rho_{1}$ into the kinetic equation (33), and recalling that the force $\dot{P}_{n}=F_{n}^{c}+\Delta X_{n}$, we get two terms: $\operatorname{Tr}\left(F_{n}^{c} \Delta \rho_{1}(t)\right)=\int F_{n}^{c} \operatorname{tr}\left(\Delta \rho_{1}\right) d \Gamma$ and $\int \operatorname{tr}\left(\Delta \rho_{1}(t) \Delta X_{n}\right) d \Gamma$. The quantum trace of $\Delta \rho_{1}$, needed for the first term, is obtained directly from Eq. (38) by using the following identities:

$$
\begin{gathered}
\operatorname{tr}\left(\rho_{\text {rel }}(r)\right)=f(P, Q, r) \\
\operatorname{tr}\left(\rho_{\text {rel }}(r) \widetilde{\Delta X}_{k}(s)\right)=f(P, Q, r) \operatorname{tr}\left(\rho_{e q} \Delta X_{k}\right)=0
\end{gathered}
$$

where the cyclic invariance of the trace was used to obtain the second identity. Thus, we obtain:

$$
\operatorname{tr}\left(\Delta \rho_{1}(t)\right)=-\int_{-\infty}^{0} d s e^{\varepsilon s} \sum_{j} \frac{\beta}{M_{j}}\left\{\left\langle P_{j}\right\rangle^{r}\left(F_{j}^{c}-\left\langle F_{j}^{c}\right\rangle_{r e l}^{r}\right)-\sum_{i}\left\langle P_{j}\right\rangle^{r} \frac{\partial\left\langle F_{j}^{c}\right\rangle_{r e l}^{r}}{\partial\left\langle Q_{i}\right\rangle^{r}}\left(Q_{i}-\left\langle Q_{i}\right\rangle^{r}\right)\right\} f(P, Q, r)
$$

and, therefore,

$$
\begin{aligned}
\operatorname{Tr}\left(F_{n}^{c} \Delta \rho_{1}(t)\right)= & -\int_{-\infty}^{0} d s e^{\varepsilon s} \sum_{j} \frac{\beta}{M_{j}}\left\langle P_{j}\right\rangle^{r}\left\{\left(\left\langle F_{n}^{c} F_{j}^{c}\right\rangle_{r e l}^{r}-\left\langle F_{n}^{c}\right\rangle_{r e l}^{r}\left\langle F_{j}^{c}\right\rangle_{r e l}^{r}\right)\right. \\
& \left.-\sum_{i} \frac{\partial\left\langle F_{j}^{c}\right\rangle_{r e l}^{r}}{\partial\left\langle Q_{i}\right\rangle^{r}}\left(\left\langle F_{n}^{c} Q_{i}\right\rangle_{r e l}^{r}-\left\langle F_{n}^{c}\right\rangle_{r e l}^{r}\left\langle Q_{i}\right\rangle^{r}\right)\right\}
\end{aligned}
$$

This latter expression can be greatly simplified by virtue of Eqs. (25) and (26):

$$
\operatorname{Tr}\left(F_{n}^{c} \Delta \rho_{1}(t)\right)=\int_{-\infty}^{0} d s e^{\varepsilon s} \sum_{j} \frac{1}{M_{j}}\left[\left\langle\frac{\partial F_{j}^{c}}{\partial Q_{n}}\right\rangle_{r e l}^{r}-\frac{\partial\left\langle F_{j}^{c}\right\rangle_{r e l}^{r}}{\partial\left\langle Q_{n}\right\rangle^{r}}\right]\left\langle P_{j}\right\rangle^{r}
$$

Note that both derivatives inside the square brackets are symmetric with respect to the permutation of their indices.

We shall now turn to the second term, $\operatorname{Tr}\left(\Delta \rho_{1} \Delta X_{n}\right)$, arising in the right hand side of the kinetic equation, and substitute $\Delta \rho_{1}$ there. Noting that the trace $\operatorname{Tr}\left(F_{j}^{c} \Delta X_{n} \rho_{r e l}\right)$ is equal to zero due to $\operatorname{tr}\left(\Delta X_{n} \rho_{e q}\right)=0$, and the fact that

$$
\int P_{j} \rho_{r e l}(r) d P_{j}=\left\langle P_{j}\right\rangle^{r} \int \rho_{r e l}(r) d P_{j}
$$

which follows from the explicit dependence of $\rho_{\text {rel }}$ on the classical momenta and Eq. (17), we obtain:

$$
\operatorname{Tr}\left(\Delta \rho_{1} \Delta X_{n}\right)=-\int_{-\infty}^{0} d s e^{\varepsilon s} \sum_{j} \frac{\beta}{M_{j}}\left\langle P_{j}\right\rangle^{r}\left(X_{n}, X_{j}(s)\right)_{r e l}^{r}
$$

where we have introduced the non-equilibrium correlation function of the fluctuation of the ionic force (cf. [14, 13]):

$$
\left(X_{n}, X_{k}(s)\right)_{r e l}^{r}=\int_{0}^{\beta}\left\langle\Delta X_{n} \widetilde{\Delta X}_{k}(i \hbar \lambda+s)\right\rangle_{r e l}^{r} d \lambda=\int d Q f(Q, r) \int_{0}^{\beta}\left\langle\Delta X_{n} \widetilde{\Delta X}_{k}(i \hbar \lambda+s)\right\rangle_{e q} d \lambda
$$

The last passage in the above formula is due to the fact that the relevant distribution average under the $\lambda$-integral depends only on the $Q$ variables since the $P$ integration can be performed directly. Combining Eqs. (39) and (41), we finally obtain the kinetic equation for the ionic momenta in the following form:

$$
\frac{d\left\langle P_{n}\right\rangle^{t}}{d t}=\left\langle F_{n}^{c}\right\rangle_{r e l}^{t}+\sum_{j} \int_{-\infty}^{0} d s e^{\varepsilon s}\left\{\left[\left\langle\frac{\partial F_{j}^{c}}{\partial Q_{n}}\right\rangle_{r e l}^{r}-\frac{\partial\left\langle F_{j}^{c}\right\rangle_{r e l}^{r}}{\partial\left\langle Q_{n}\right\rangle^{r}}\right]-\beta\left(X_{n}, X_{j}(s)\right)_{r e l}^{r}\right\} \frac{\left\langle P_{j}\right\rangle^{r}}{M_{j}}
$$


This is the desired equation of motion for ions. In the right hand side it contains the total force acting on ion $n$ due to other ions. Their interaction and energy exchange with all the electrons are also completely accounted for. We also note that we have not made any assumptions as to whether electronic subsystem is in its ground state, it is in general a weighted sum of the ground and excited electronic states (see also below). In other words, this description goes beyond the adiabatic approximation.

The first term in the right hand side of Eq. (43) gives the conservative force corresponding to ionic positions at time $t$ :

$$
\left\langle F_{n}^{c}\right\rangle_{r e l}^{t}=\int F_{n}^{c}(Q) f(Q, t) d Q
$$

The second term in the right hand side of Eq. (43) gives a correction arising due to fluctuation of the ionic force. Similarly to the friction force acting on a Brownian particle immersed in a liquid, this force appears to be linear with the ions momenta. Indeed, the correlation function and derivatives inside the square brackets do not depend on the expectation values $\left\langle P_{j}\right\rangle^{t}$ since the $P$ integration in those terms can be performed explicitly and all the Lagrange multipliers $\left\{V_{j}\right\}$ disappear exactly. Moreover, the derived friction is non-Markovian, i.e. includes memory effects.

Thus, we conclude, the rigorous non-equilibrium statistical mechanical treatment of a system composed of ions and electrons results in Newton-like equations of motion for average ionic momenta that additionally contain friction forces due to energy exchange with the electronic subsystem maintained at the given temperature $T$.

The obtained equations are very complicated because of the relevant distribution used in the right hand side which depends on the observables $\left\langle P_{j}\right\rangle^{t}$ and $\left\langle Q_{j}\right\rangle^{t}$ in a rather complicated way. In the next subsection a reasonable approximation will be offered which results in a significant simplification of these equations.

\subsection{Saddle-point approximation}

Let us consider a relevant distribution average of some function, $\zeta(Q)$, depending only on classical coordinates:

$$
\langle\zeta(Q)\rangle_{r e l}^{t}=\int \zeta(Q) f(Q, t) d Q
$$

where the $Q$-distribution is given explicitly by Eq. (16). Let $\left\{\psi_{m}(q, Q) ; m=0,1,2, \ldots\right\}$ is the complete set of electronic wavefunctions, depending parametrically on the positions of ions $Q$. The wavefunctions $\psi_{m}$ are the eigenvectors of the electronic Hamiltonian, i.e. $\mathcal{H}_{q} \psi_{m}=\left(\varepsilon_{m}-\mu\right) \psi_{m}$. Then, it is easily seen that the "partition function" of the $Q$-distribution, $f(Q, t)$, can be written as a sum:

$$
Z_{Q}=\int e^{-\beta \Re(Q)}\left(1+\sum_{m=1}^{\infty} e^{-\beta \Delta \varepsilon_{m}(Q)}\right) d Q
$$

where

$$
\Re(Q)=\varepsilon_{0}(Q)-\mu+U(Q)+\sum_{j}\left\langle F_{j}^{c}\right\rangle_{r e l}^{t} Q_{j}
$$

and $\Delta \varepsilon_{m}(Q)=\varepsilon_{m}(Q)-\varepsilon_{0}(Q)$ are exact electronic excitation energies for the given geometry of the nuclei, $Q$. We assume hereafter that the ground state is non-degenerate for any geometry, and thus all the excitation energies are strictly positive. Moreover, we assume that for any geometry $Q$ there is a gap between the ground and the first excited states, and the ground state energy in the external field, $\varepsilon_{0}(Q)+U(Q)$, has a minimum at some geometry $Q_{0}$. Of course, the minimum will be affected by the last term in Eq. (46), however, we assume that this term does not change significantly the potential energy surface of the ground state. Therefore, the function $\Re(Q)$ will still have a minimum at some geometry $Q^{t}$ (the subscript reflects the fact that, because of the last term in Eq. (46), the minimum geometry $Q^{t}$ will depend on time), and thus can be expanded in a series with respect to the difference $Q-Q^{t}:$

$$
\Re(Q)=\Re\left(Q^{t}\right)+\frac{1}{2} \sum_{i j}\left(\frac{\partial^{2} \Re}{\partial Q_{i} \partial Q_{j}}\right)_{Q^{t}}\left(Q_{i}-Q_{i}^{t}\right)\left(Q_{j}-Q_{j}^{t}\right)+\ldots
$$

where the matrix $\left\|\left(\frac{\partial^{2} \Re}{\partial Q_{i} \partial Q_{j}}\right)_{Q^{t}}\right\|$ of second derivatives is positively defined (since $Q^{t}$ is the minimum). Hence, the function $e^{-\beta \Re(Q)}$ will be highly peaked around $Q^{t}$, whereas the function in the round brackets in Eq. (45) can be assumed to be a rather slowly changing with $Q$ and can thus be taken away from the $Q$-integral with all ionic positions calculated at $Q \equiv Q^{t}$. A simple calculation in the spirit of the well-known saddle-point approximation will then show that the distribution function $f(Q, t)$ effectively serves as a Delta function $\delta\left(Q-Q^{t}\right)$ giving for the 
average of any slowly changing function of ionic positions, $\zeta(Q)$, the following simple result: $\langle\zeta(Q)\rangle_{\text {rel }}^{t} \simeq \zeta\left(Q^{t}\right)$. Further, if we consider specifically $\zeta(Q) \equiv Q_{j}$, then we obtain that $\left\langle Q_{j}\right\rangle^{t}=\left\langle Q_{j}\right\rangle_{\text {rel }}^{t}$ should be replaced with $Q_{j}^{t}$. Thus, we conclude that in a consistent application of the saddle point approximation, one replaces the averages $\langle\zeta(Q)\rangle_{\text {rel }}^{t}$, calculated with respect to the $Q$-distribution, with the corresponding functions $\zeta$ calculated at exact average ionic positions at time $t$, namely:

$$
\langle\zeta(Q)\rangle_{r e l}^{t} \simeq \zeta\left(\langle Q\rangle^{t}\right)
$$

In particular, this result can be applied to the non-equilibrium correlation function of Eq. (42) resulting in a much simpler expression for it:

$$
\left(X_{n}, X_{k}(s)\right)_{r e l}^{t} \simeq\left[\int_{0}^{\beta}\left\langle\Delta X_{n} \widetilde{\Delta X}_{k}(i \hbar \lambda+s)\right\rangle_{e q} d \lambda\right]_{Q=\langle Q\rangle^{t}}=\left(X_{n}, X_{k}(s)\right)_{e q}^{\langle Q\rangle^{t}}
$$

Thus, the correlation function depends directly on a single time $s$; however, the statistical average is to be calculated over the equilibrium distribution $\rho_{e q}$ corresponding to ions fixed in their exact positions $\langle Q\rangle^{t}$ at another time $t$.

Using the same approach, one can also verify that the term in the square brackets in the right hand side of the equation of motion (43) can be dropped. Indeed, since the conservative forces $F_{j}^{c}$ depend entirely on ionic positions, we can write:

$$
\left\langle\frac{\partial F_{j}^{c}}{\partial Q_{n}}\right\rangle_{r e l}^{r} \simeq\left(\frac{\partial F_{j}^{c}}{\partial Q_{n}}\right)_{Q=\langle Q\rangle^{r}}=\frac{\partial F_{j}^{c}\left(\langle Q\rangle^{r}\right)}{\partial\left\langle Q_{n}\right\rangle^{r}}
$$

and, at the same time,

$$
\frac{\partial\left\langle F_{j}^{c}\right\rangle_{r e l}^{r}}{\partial\left\langle Q_{n}\right\rangle^{r}} \simeq \frac{\partial F_{j}^{c}\left(\langle Q\rangle^{r}\right)}{\partial\left\langle Q_{n}\right\rangle^{r}}
$$

Therefore, within the saddle-point approximation, the difference of derivatives in the square brackets in the equation of motion (43) is equal to zero. Following the same arguments and replacing the conservative force $\left\langle F_{n}^{c}\right\rangle_{\text {rel }}^{t}$ in the equation of motion with $F_{n}^{c}\left(\langle Q\rangle^{t}\right)$, we obtain:

$$
\frac{d\left\langle P_{n}\right\rangle^{t}}{d t}=F_{n}^{c}\left(\langle Q\rangle^{t}\right)-\sum_{j} \frac{\beta}{M_{j}} \int_{-\infty}^{0} d s e^{\varepsilon s}\left(X_{n}, X_{j}(s)\right)_{e q}^{\langle Q\rangle^{t}}\left\langle P_{j}\right\rangle^{r}
$$

which is the final result.

We see that, if not for the friction term, the equations of motion would have corresponded exactly to the Newton's equations of motion for ions: in the left hand side we have the time derivative of ion $n$ momentum, while in the right hand side - the total statistically averaged force acting on this ion at the given temperature:

$$
\begin{gathered}
F_{n}^{c}\left(\langle Q\rangle^{t}\right)=\left\langle-\frac{\partial H}{\partial Q_{n}}\right\rangle_{e q}^{Q=\langle Q\rangle^{t}}=-\frac{\partial U}{\partial Q_{n}}+\left[Z_{e q}^{-1} \sum_{m} e^{-\beta\left(\varepsilon_{m}-\mu\right)}\left\langle\psi_{m}\left|-\frac{\partial H_{q}}{\partial Q_{n}}\right| \psi_{m}\right\rangle\right]_{Q=\langle Q\rangle^{t}} \\
=-\frac{\partial U}{\partial Q_{n}}+\left[Z_{e q}^{-1} \sum_{m} e^{-\beta\left(\varepsilon_{m}-\mu\right)}\left(-\frac{\partial \varepsilon_{m}}{\partial Q_{n}}\right)\right]_{Q=\langle Q\rangle^{t}}
\end{gathered}
$$

where $Z_{e q}=\sum_{m} \exp \left(-\beta\left(\varepsilon_{m}-\mu\right)\right)$. Here, $-\frac{\partial \varepsilon_{m}}{\partial Q_{n}}$ is the force acting on ion $n$ (due to electrons and nuclei) when the electronic subsystem is in electronic state $m$ (i.e. on the adiabatic potential energy surface, $\varepsilon_{m}(Q)$, corresponding to electronic state $m$ ).

Note that atomic positions, $\langle Q\rangle^{t}$, correspond exactly to the averaged ionic momenta, $\langle P\rangle^{t}$, see Eq. (32). The obtained equations of motion are more general than those of ordinary Molecular Dynamics. Indeed, in standard MD the forces do not depend on temperature and are calculated as in Eq. (50) from the conservative part taking only the electronic ground state into account,

$$
F_{n}^{c} \simeq-\frac{\partial U}{\partial Q_{n}}+\left\langle\psi_{0}\left|-\frac{\partial H_{q}}{\partial Q_{n}}\right| \psi_{0}\right\rangle=-\frac{\partial\left(U+\varepsilon_{0}\right)}{\partial Q_{n}}
$$

(the Car-Parinello ground state $a b$ initio MD simulations [4] being an obvious example). Therefore our equations may serve as a justification of MD simulations which go beyond the Born-Oppenheimer approximation (see, e.g. [11]), where, when calculating the force acting on an ion, it is assumed explicitly that the electronic subsystem may 
occupy both ground and excited electronic states. In fact, in complete agreement with the principles of quantum statistical mechanics, we show that there is a certain probability for the electronic subsystem to occupy every state at the same time, which, one must admit, is somewhat different from most of the non-adiabatic computational techniques [6] in which it is usually assumed that only one state can be occupied at every single time step.

We also observe that a consistent non-equilibrium treatment results in additional terms in the equations of motion which are proportional to the ions momenta and thus have the meaning of friction, related to the energy exchange between the ions and the electrons; the latter serving as a "thermostat" held at a given temperature. Thus, our rigorous treatment justifies the usage of "electronic friction" terms in MD simulations [11] and explains their physical origin.

\subsection{Non-equilibrium correlation functions}

Since the operator of the atomic force, $X_{i}=-\frac{\partial \Phi}{\partial Q_{i}}=\sum_{k=1}^{N} x_{i}\left(\mathbf{r}_{k}\right)$, is a derivative of the electron-phonon interaction energy, $\Phi(q, Q)=\sum_{k=1}^{N} \phi\left(\mathbf{r}_{k}, Q\right)$, it is a one-particle operator,

$$
X_{i}=\sum_{a b} X_{a b}^{i} c_{a}^{\dagger} c_{b}
$$

(where $c_{a}^{\dagger}$ and $c_{b}$ are creation and annihilation operators in some basis set of spin-orbitals) and $X_{a b}^{i}=\left\langle a\left|x_{i}(\mathbf{r})\right| b\right\rangle$. Here $x_{i}(\mathbf{r})=-\frac{\partial \phi(\mathbf{r}, Q)}{\partial Q_{i}}$ is the force on atom $i$ due to a single electron at $\mathbf{r}, \phi(\mathbf{r}, Q)$ being the interaction energy of this electron with all nuclei. Hence in general, the correlation function (48) is a two-particle equilibrium statistical average, containing four $c$-operators, and thus cannot be calculated exactly in the general case.

The calculation is straightforward if the Hamiltonian $H_{q}=\sum_{a b} h_{a b} c_{a}^{\dagger} c_{b}$ is a one-particle operator (e.g. in the Hartree-Fock approximation). Indeed, in this case one can diagonalise the Hamiltonian,

$$
H_{q}=\sum_{\sigma} \xi_{\sigma} d_{\sigma}^{\dagger} d_{\sigma}
$$

where

$$
d_{\sigma}=\sum_{a} e_{\sigma a}^{*} c_{a}, \quad d_{\sigma}^{\dagger}=\sum_{a} e_{\sigma a} c_{a}^{\dagger}
$$

and $\xi_{\sigma}$ and $e_{\sigma}=\left\|e_{\sigma a}\right\|$ are the eigenvalues and the eigenvectors of the matrix $h=\left\|h_{a b}\right\|$, and therefore express the operators $X_{i}$ and $X_{j}$ via the operators $d_{\sigma}^{\dagger}$ and $d_{\sigma}$, e.g.

$$
X_{i}=\sum_{\sigma \sigma^{\prime}} x_{\sigma \sigma^{\prime}}^{(i)} d_{\sigma}^{\dagger} d_{\sigma^{\prime}}, x_{\sigma \sigma^{\prime}}^{(i)}=\sum_{a b} X_{a b}^{i} e_{\sigma a}^{*} e_{\sigma^{\prime} b}
$$

Since the calculation of the operators $\widetilde{d}_{\sigma}^{\dagger}(t)$ and $\widetilde{d}_{\sigma}(t)$ in the Heisenberg representation is simple, we obtain:

$$
\left(X_{i}, X_{j}(t)\right)_{e q}=\sum_{\sigma \sigma^{\prime}} x_{\sigma \sigma^{\prime}}^{(i)} x_{\sigma^{\prime} \sigma}^{(j)} n_{\sigma}\left(1-n_{\sigma^{\prime}}\right) \chi\left(\xi_{\sigma^{\prime}}-\xi_{\sigma}\right) e^{i\left(\xi_{\sigma^{\prime}}-\xi_{\sigma}\right) t / \hbar}
$$

where $n_{\sigma}=\left(e^{\beta\left(\xi_{\sigma}-\mu\right)}+1\right)^{-1}$ and $\chi(E)=E^{-1}\left(1-e^{-\beta E}\right)$ if $E \neq 0$ and $\chi(0)=\beta$. Note that the superscript $\langle Q\rangle^{t}$ to the correlation function has been omitted to simplify the notations.

When the electron-electron interaction is accounted for explicitly, one has to develop more powerful methods. First we note that the correlation function, Eq. (48), may be considered as a particular case of a more general correlation function defined for any two quantum operators $A$ and $B$ as follows:

$$
\left(A\left(t_{1}\right), B\left(t_{2}\right)\right)_{e q}=\int_{0}^{\beta} d \lambda \operatorname{tr}\left[\widetilde{A}\left(t_{1}\right) \widetilde{B}\left(t_{2}+i \lambda \hbar\right) \rho_{e q}\right] \equiv \int_{0}^{\beta} d \lambda\left\langle\widetilde{A}\left(t_{1}\right) \widetilde{B}\left(t_{2}+i \lambda \hbar\right)\right\rangle_{e q}
$$

This correlation function obeys some simple symmetry properties:

$$
\left(A\left(t_{1}\right), B\left(t_{2}\right)\right)_{e q}=\left(A, B\left(t_{2}-t_{1}\right)\right)_{e q}=\left(A\left(t_{2}\right), B\left(t_{1}\right)\right)_{e q}
$$

which follow from the cyclic invariance of the trace (change of variables $\lambda \rightarrow \lambda_{1}=\beta-\lambda$ is also necessary to obtain the last equality). 
An explicit expression for the correlation function can be obtained using the complete set, $\left\{\psi_{n}\right\}$, of the eigenvectors of the electronic Hamiltonian (i.e. $H_{q} \psi_{n}=\varepsilon_{n} \psi_{n}$ ):

$$
(A, B(t))_{e q}=\sum_{n m} \rho_{n} \chi\left(E_{m}-E_{n}\right)\left\langle\psi_{n}|A| \psi_{m}\right\rangle\left\langle\psi_{m}|B| \psi_{n}\right\rangle e^{i\left(\varepsilon_{m}-\varepsilon_{m}\right) t / \hbar}
$$

where $\rho_{n}=Z_{e q}^{-1} e^{-\beta\left(\varepsilon_{n}-\mu N\right)}$. It is also convenient to introduce the spectral function (matrix) as the Fourier transform of the correlation function:

$$
(A, B(t))_{e q}=\frac{1}{2 \pi} \int_{-\infty}^{\infty} e^{i \omega t} J_{A B}(\omega) d \omega
$$

where

$$
J_{A B}(\omega)=2 \pi \hbar \sum_{n m} \rho_{n} \chi\left(E_{m}-E_{n}\right)\left\langle\psi_{n}|A| \psi_{m}\right\rangle\left\langle\psi_{m}|B| \psi_{n}\right\rangle \delta\left(\varepsilon_{m}-\varepsilon_{n}-\hbar \omega\right)
$$

which satisfy the following symmetry properties:

$$
J_{A B}(\omega)=J_{B A}(-\omega)=J_{B A}(\omega)^{*}
$$

Unfortunately, it appears impossible to develop systematically a perturbation theory for the direct calculation of the correlation function (54). It has been found, however, that such a perturbation theory exists for the equilibrium statistical average inside the $\lambda$-integral, i.e for the $\left\langle\widetilde{A}\left(t_{1}\right) \widetilde{B}\left(t_{2}+i \lambda \hbar\right)\right\rangle_{e q}$. Once the statistical average is calculated, the correlation function follows immediately. The method is based on a relationship between the correlation function above and the Matsubara Green's functions (e.g. [22, 23]); this is revealed in Appendix. Therefore, one can use the perturbation expansion (and thus the powerful diagrammatic techniques) to account for the electron-electron interactions in calculating the correlation function appearing in Eq. (48).

\subsection{Perturbative solution of the Liouville equation: second order}

The formulae developed in the previous Sections correspond to the first order approximation as we kept only terms of the order of $M^{-1 / 2}$. This treatment can be systematically extended to higher orders. Unfortunately, this results in very cumbersome expressions even in the second order. Therefore, in this Section we shall simply outline the main idea of how the extension to higher orders can be done.

We start from Eq. (34) and replace the exponential operator there with its expansion given by Eq. (37). After that, we recall that the operator $\left(\frac{\partial}{\partial r}+i \widehat{L}\right) \rho_{\text {rel }}(r)$ contains the trace of $\Delta \rho$ as well (see Eq. (36) $)$ and thus this dependence must also be included in developing the perturbative expansion. For instance, one gets for the next order term:

$$
\begin{aligned}
\Delta \rho_{2}(t) & =-\int_{-\infty}^{0} d s e^{\varepsilon s} \sum_{j} \frac{\beta}{M_{j}}\left(P_{j}-\left\langle P_{j}\right\rangle^{r}\right) \rho_{r e l}(r) \operatorname{Tr}\left(\dot{P}_{j} \Delta \rho_{1}(r)\right) \\
& -\int_{-\infty}^{0} d s e^{\varepsilon s} \int_{0}^{1} d x e^{i x s \widehat{L}_{q}}\left(i s \widehat{L}_{c}\right) e^{-i x s \widehat{L}_{q}} e^{-i s \widehat{L}_{q}} \widehat{A}
\end{aligned}
$$

where $\widehat{A}$ is obtained by removing the trace term in the operator $\left(\frac{\partial}{\partial r}+i \widehat{L}\right) \rho_{\text {rel }}(r)$ (see Eqs. (35) and (36)) and $\Delta \rho_{1}$ is the first order term given by Eq. (38).

First of all, it can easily be shown that $\operatorname{Tr}\left(\Delta \rho_{2}\right)=0$, i.e. this correction is consistent as well with the correct normalisation of the statistical operator. Indeed, the trace of the first term in Eq. (60) vanishes due to integration over $P_{j}$ in the classical part of the trace and Eq. (40). The second term also does not contribute to the trace of $\Delta \rho_{2}$ due to the cyclic invariance of the trace and the operator identity (10) (the operator $\widehat{A}$ is proportional to the relevant distribution and thus vanishes at the boundaries of the classical phase space).

It can also be shown that any order correction to the statistical operator, $\Delta \rho_{n}$, has zero trace and hence does not break down the normalisation of the statistical operator.

Using the explicit expression for $\Delta \rho_{2}$ given above, one can calculate its contribution to the force, $\operatorname{Tr}\left(\dot{P}_{n} \Delta \rho_{2}(t)\right)$, in the right-hand side of the equations of motion (33). The contribution is very cumbersome (and is only due to the second term in Eq. (60) ) and won't be reproduced here. We only note that it is proportional to the square of the atomic momenta, i.e. it contains terms proportional to $\left\langle P_{j}\right\rangle^{r}\left\langle P_{k}\right\rangle^{r}$. Higher order terms contain more products of the atomic momenta. Also, much more complicated correlation functions appear as the kernels of the time integrals in the contribution to the force. 


\section{Conclusions}

In this paper we have considered, using intrinsically non-equilibrium statistical mechanical theory, a system of fast (electrons) and slow (atoms) particles which interact with each other and may interchange their energy. The system is enclosed in a thermal bath kept at a constant temperature. In our treatment, the combined quantum-classical consideration was used: the slow degrees of freedom (atomic coordinates) were treated classically, while the fast variables (electrons) quantum mechanically. No assumption was made concerning the structure of the statistical operator; in particular, it is not in any way factorised. In addition, the electronic subsystem was treated exactly with complete inclusion of electron-electron interaction.

We show, by assuming that the classical degrees of freedom are much "heavier" than the quantum ones, that equations of motion for the former (i.e for atoms) contain the conservative and friction forces. The conservative forces are statistically averaged over the electronic states. The friction force, which is strictly proportional to the atoms momenta, is expressed via the correlation function of the fluctuating force with which electrons act on the atoms (i.e. due to the fluctuation of the electron-phonon interaction). The correlation function can be expressed via two-electron Matsubara Green's function and thus calculated using the well-developed perturabtion diagrammatic techniques.

The theory presented here gives a solid foundation for a number of intuitive theories based on MD simulations which go beyond the Born-Oppenheimer approximation (see, e.g. [11, 6]). We also justify the usage of "electronic friction" terms in MD simulations [1] and explain their physical origin.

In our method it was assumed that on average the electronic subsystem is in thermodynamic equilibrium. For instance, the theory developed here is applicable to ordinary Molecular Dynamics when atoms move along classical trajectories. However, the theory can also be applied in other cases in which the electronic subsystem is in a steady state, i.e. which, on average (more precisely, over the timescale associated with atomic motion), is not time dependent (is stationary), for instance, stationary electronic or heat conductance in an atomic wire [9, 6].

A number of avenues exist in developing our theory further. Firstly, as has been mentioned above, we have assumed in our treatment that electrons quickly reach thermodynamic equilibrium during the motion of atoms. In some cases this assumption will not be valid, e.g. when considering non-elastic effects during a nonstationary conductance in a system. In these and similar cases one has to include additional relevant variables into the consideration, e.g. time dependent electronic density matrix as in the kinetic theories [12]. This approach would result in an additional kinetic equation for the density to be solved simultaneously with the atomic motion considered here. Secondly, another possible extention is concerned with considering nuclei quantum mechanically as well. This, however, is much more difficult and may be done by e.g. starting from the method developed in Ref. [9].

\section{Acknowledgemnents}

Yu Wang would like to acknowledge the financial support from the China Scholarship Council, The K. C. Wong Education Foundation (Hong Kong), The Henry Lester Trust Limited and The Leche Trust, which made this work possible. We would also like to thank Tchavdar Todorov, Andrew Horsefield and Mike Finnis for stimulating discussions and useful suggestions.

\section{Appendix}

In this Appendix we shall relate the correlation function (48) with the thermodynamic Matsubara Green's function (e.g. [22, 23]). We shall start by defining a "complex time" Green's function for two arbitrary operators $A$ and $B$ as follows:

$$
G_{A B}\left(x_{1}, x_{2}\right)=-\operatorname{tr}\left[\rho_{e q} \widehat{T}_{x}\left(\bar{A}\left(x_{1}\right) \bar{B}\left(x_{2}\right)\right)\right]
$$

where the quantum operators $A$ and $B$ appearing in the Green's function are written in the special representation defined with respect to a single real parameter $x$. This specific representation which will be designated in what follows with the bar over the operator symbol, is given by

$$
\bar{A}(x)=e^{i \tau(x) \mathcal{H}_{q} / \hbar} A e^{-i \tau(x) \mathcal{H}_{q} / \hbar}
$$

where the complex "time" $\tau(x)=\zeta x$ is introduced which contains the complex prefactor $\zeta=-(t+i \lambda \hbar) / \lambda$ and changes linearly with $x$, so that $\tau(0)=0$ and $\tau(-\lambda)=t+i \lambda \hbar$. The operator $\widehat{T}_{x}$ performs chronological ordering of the operators $\bar{A}\left(x_{1}\right)$ and $\bar{B}\left(x_{2}\right)$, so that $x$ increases from right to left:

$$
\widehat{T}_{\lambda}\left(\bar{A}\left(x_{1}\right) \bar{B}\left(x_{2}\right)\right)=\theta\left(x_{1}-x_{2}\right) \bar{A}\left(x_{1}\right) \bar{B}\left(x_{2}\right)+\eta \theta\left(x_{2}-x_{1}\right) \bar{B}\left(x_{2}\right) \bar{A}\left(x_{1}\right)
$$


where $\eta= \pm 1$ corresponds to the sign acquired when changing the order of the operators $\bar{A}\left(x_{1}\right)$ and $\bar{B}\left(x_{2}\right) ; \theta(x)$ is the Heviside step function.

Using the cyclic invariance of the trace, it is easy to see that the Green's function (61) actualy depends only on the difference $x=x_{1}-x_{2}$ :

$$
G_{A B}\left(x_{1}, x_{2}\right) \equiv G_{A B}(x)=-\theta(x)\langle A \bar{B}(-x)\rangle_{e q}-\eta \theta(-x)\langle\bar{B}(-x) A\rangle_{e q}
$$

The correlation function we would like to calculate, $\langle A \widetilde{B}(t+i \lambda \hbar)\rangle_{e q}$ with $0<\lambda<\beta$, is equal directly to the minus Green's function $G_{A B}(0,-\lambda) \equiv G_{A B}(\lambda)$. Moreover, using eigenvectors and eigenvalues of the Hamiltonian, $\mathcal{H}_{q} \psi_{n}=\left(\varepsilon_{n}-\mu N\right) \psi_{n}$, one obtains:

$$
\begin{gathered}
\langle A \bar{B}(-x)\rangle_{e q}=\sum_{n m} \rho_{n}\left\langle\psi_{n}|A| \psi_{m}\right\rangle\left\langle\psi_{m}|B| \psi_{n}\right\rangle e^{i\left(\varepsilon_{m}-\varepsilon_{n}\right) x t / \lambda \hbar} e^{-x\left(\epsilon_{m}-\epsilon_{n}\right)}, x>0 \\
\langle\bar{B}(-x) A\rangle_{e q}=\sum_{n m} \rho_{n}\left\langle\psi_{n}|A| \psi_{m}\right\rangle\left\langle\psi_{m}|B| \psi_{n}\right\rangle e^{i\left(\varepsilon_{m}-\varepsilon_{n}\right) x t / \lambda \hbar} e^{-(x+\beta)\left(\epsilon_{m}-\epsilon_{n}\right)}, x<0
\end{gathered}
$$

where $\rho_{n}=Z_{e q}^{-1} e^{-\beta\left(\epsilon_{n}-\mu N\right)}$. It is easily verified (by splitting each double sum into two contributions with positive energy differences each) that the first correlation function, Eq. (64), converges for $x<\beta$ (and $x>0$ ), while the second one for $x>-\beta$ (and $x<0$ ). Therefore, $x$ is limited to the interval $-\beta<x<\beta$.

Note also that the above correlation functions can also be written as Fourier integrals:

$$
\begin{gathered}
G_{A B}(x)=-\langle A \bar{B}(-x)\rangle_{e q}=\int_{-\infty}^{\infty} \frac{d \omega}{2 \pi} I_{A B}(\omega) e^{i \omega x t / \lambda} e^{-x \omega \hbar}, \quad x>0 \\
G_{A B}(x)=-\eta\langle\bar{B}(-x) A\rangle_{e q}=\eta \int_{-\infty}^{\infty} \frac{d \omega}{2 \pi} I_{A B}(\omega) e^{i \omega x t / \lambda} e^{-(x+\beta) \omega \hbar}, \quad x<0
\end{gathered}
$$

where the spectral function

$$
I_{A B}(\omega)=-2 \pi \sum_{n m} \rho_{n}\left\langle\psi_{n}|A| \psi_{m}\right\rangle\left\langle\psi_{m}|B| \psi_{n}\right\rangle \delta\left(\frac{\epsilon_{m}-\epsilon_{n}}{\hbar}-\omega\right)
$$

So far, the Green's function introduced above in Eq. (61) has been shown to possess properties very similar or even identical to those of the Matsubara Green's function [22, 23]. To strengthen this analogy, one can also expand the Green's function into a Fourier series in the interval $-\beta<x<\beta$ or notice that the two Green's functions, for $-\beta<x<0$ and $0<x<\beta$, make a jump at $x=0$ :

$$
\lim _{\delta \rightarrow+0}\left[G_{A B}(\delta)-G_{A B}(-\delta)\right]=\int_{-\infty}^{\infty} \frac{d \omega}{2 \pi} I_{A B}(\omega)\left(1-\eta e^{-\beta \hbar \omega}\right)
$$

However, this analogy is not complete; for instance, one can see from the integral representations of the Green's function given above that $G_{A B}(x)$ for $x<0$ is not related to the $G_{A B}(x+\beta)$ although they both share the same spectral function $I_{A B}(\omega)$.

At this point we are quite prepared to find the relationship between the "complex time" and the Matsubara Green's functions. To this end, we shall first introduce the appropriate "interaction representation" for the operators:

$$
\bar{A}_{I}(x)=e^{i \tau(x) \mathcal{H}_{0} / \hbar} A e^{-i \tau(x) \mathcal{H}_{0} / \hbar}
$$

where $\mathcal{H}_{0}$ is the Hamiltonian of non-interacting electrons, i.e. $\mathcal{H}_{q}=\mathcal{H}_{0}+H^{\prime}$ with $H^{\prime}$ being the electron-electron interaction. Then, the product of two operators in the Green's function (61) can be written as

$$
\bar{A}\left(x_{1}\right) \bar{B}\left(x_{2}\right)=U\left(0, x_{1}\right) \bar{A}_{I}\left(x_{1}\right) U\left(x_{1}, x_{2}\right) \bar{B}_{I}\left(x_{2}\right) U\left(x_{2}, 0\right)
$$

where

$$
U\left(x_{1}, x_{2}\right)=e^{i \tau\left(x_{1}\right) \mathcal{H}_{0} / \hbar} e^{-i\left(\tau\left(x_{1}\right)-\tau\left(x_{2}\right)\right) \mathcal{H}_{q} / \hbar} e^{-i \tau\left(x_{2}\right) \mathcal{H}_{0} / \hbar}
$$


is the evolution operator in our interaction representation. The evolution operator defined above satisfies the differential equation $\left(\kappa=\frac{i \zeta}{\hbar}=1-\frac{i t}{\hbar \lambda}\right)$

$$
\frac{\partial U\left(x_{1}, x_{2}\right)}{\partial x_{1}}=-\kappa \bar{H}_{I}^{\prime}\left(x_{1}\right) U\left(x_{1}, x_{2}\right)
$$

which can be converted into an integral equation and then solved iteratively, giving as a solution:

$$
U\left(x_{1}, x_{2}\right)=\widehat{T}_{x} \exp \left(-\kappa \int_{x_{2}}^{x_{1}} \bar{H}_{I}^{\prime}(x) d x\right)
$$

Thus, the evolution operator can be expressed via the $T_{x}$-exponent. Note that $\kappa$ actually depends on the particular values of $t$ and $\lambda$ used to define the "complex times" in the Green 's function arguments.

The fact that the evolution operator can be expressed via the chronologically ordered exponent allows for the considerable simplification of the chronological product of the two operators in the Green's function. Indeed, the product in Eq. (61) can be rewritten as

$$
\begin{gathered}
\widehat{T}_{x}\left(\bar{A}\left(x_{1}\right) \bar{B}\left(x_{2}\right)\right)=\widehat{T}_{x}\left(U\left(0, x_{1}\right) \bar{A}_{I}\left(x_{1}\right) U\left(x_{1}, x_{2}\right) \bar{B}_{I}\left(x_{2}\right) U\left(x_{2}, 0\right)\right) \\
=\widehat{T}_{x}\left(U\left(0, x_{1}\right) U\left(x_{1}, x_{2}\right) U\left(x_{2}, 0\right) \bar{A}_{I}\left(x_{1}\right) \bar{B}_{I}\left(x_{2}\right)\right) \\
=\widehat{T}_{x}\left(U(0,0) \bar{A}_{I}\left(x_{1}\right) \bar{B}_{I}\left(x_{2}\right)\right)=\widehat{T}_{x}\left(\bar{A}_{I}\left(x_{1}\right) \bar{B}_{I}\left(x_{2}\right)\right)
\end{gathered}
$$

where use has been made of the obvious properties of the evolution operator: $U(x, x)=1$ and $U\left(x_{1}, x_{2}\right) U\left(x_{2}, x_{3}\right)=$ $U\left(x_{1}, x_{3}\right)$. Thus, we see that the electron-electron interaction can be actually eliminated entirely from the chronological product of the operators in the Green's function.

Although the method developed below is valid for any operators $A$ and $B$, we shall consider the particular case needed here when the operators are of the binary form given by Eq. (52) (note also that $\eta=1$ in this case). To proceed, we recognise that the creation and annihilation operators, $d_{\sigma}^{\dagger}$ and $d_{\sigma}$ (in the representation that diagonalises the Hamiltonian $\mathcal{H}_{0}$, see Section 3.5), have a very simple form both in the standard thermodynamic (imaginary time) and our (complex time) representations. To simplify the notations, we shall use from now on in this Appendix a wavy line above operators for the usual thermodynamic interaction representation of the operators, i.e. $\widetilde{C}_{I}(x)=e^{x \mathcal{H}_{0}} C e^{-x \mathcal{H}_{0}}$. Then, one has:

$$
\begin{gathered}
\bar{d}_{\sigma I}(x)=d_{\sigma} e^{-i \tau(x) \xi_{\sigma} / \hbar}, \bar{d}_{\sigma I}^{\dagger}(x)=d_{\sigma}^{\dagger} e^{i \tau(x) \xi_{\sigma} / \hbar} \\
\widetilde{d}_{\sigma I}(x)=e^{x \mathcal{H}_{0}} d_{\sigma} e^{-x \mathcal{H}_{0}}=d_{\sigma} e^{-x \xi_{\sigma}}, \widetilde{d}_{\sigma I}^{\dagger}(x)=d_{\sigma}^{\dagger} e^{x \xi_{\sigma}}
\end{gathered}
$$

Therefore, any binary operator, $A=\sum_{\sigma \sigma^{\prime}} A_{\sigma \sigma^{\prime}} d_{\sigma}^{\dagger} d_{\sigma^{\prime}}$, when written in the "complex time" interaction representation, $\bar{A}_{I}(x)$, can easily be expressed as another operator $A^{\prime}$ written in the ordinary thermodynamic representation, i.e.

$$
\bar{A}_{I}(x)=\sum_{\sigma \sigma^{\prime}} A_{\sigma \sigma^{\prime}} \bar{d}_{\sigma}^{\dagger}(x) \bar{d}_{\sigma^{\prime}}(x)=\sum_{\sigma \sigma^{\prime}} A_{\sigma \sigma^{\prime}}^{\prime}(x) \widetilde{d}_{\sigma}^{\dagger}(x) \widetilde{d}_{\sigma^{\prime}}(x) \equiv \widetilde{A}_{I}^{\prime}(x)
$$

with the new matrix of the coefficients $\mathbf{A}^{\prime}(x)=\left\|e^{(\kappa-1) x\left(\xi_{\sigma}-\xi_{\sigma^{\prime}}\right)} A_{\sigma \sigma^{\prime}}\right\|$ which depends explicitly on $x$. This simple result allows us to rewrite the chronological product of the operators of Eq. (69) as

$$
\widehat{T}_{x}\left(\bar{A}\left(x_{1}\right) \bar{B}\left(x_{2}\right)\right)=\widehat{T}_{x}\left(\bar{A}_{I}\left(x_{1}\right) \bar{B}_{I}\left(x_{2}\right)\right)=\widehat{T}_{x}\left(\widetilde{A}_{I}^{\prime}\left(x_{1}\right) \widetilde{B}_{I}^{\prime}\left(x_{2}\right)\right)
$$

In the final expression above the product of two operators appears exactly as in the thermodynamic (Matsubara) Green's function. To finish the transformation, we should introduce the evolution operator in the usual thermodynamic representation:

$$
\widetilde{U}\left(x_{1}, x_{2}\right)=e^{x_{1} \mathcal{H}_{0}} e^{-\left(x_{1}-x_{2}\right) \mathcal{H}_{q}} e^{-x_{2} \mathcal{H}_{0}} \equiv \widehat{T}_{x} \exp \left[-\int_{x_{2}}^{x_{1}} \widetilde{H}_{I}^{\prime}(x) d x\right]
$$

that satisfies the properties $\widetilde{U}\left(x_{1}, x_{2}\right) \widetilde{U}\left(x_{2}, x_{3}\right)=\widetilde{U}\left(x_{1}, x_{3}\right)$ and $\widetilde{U}(x, x)=1$. The evolution operator enters the equilibrium statistical operator, $\rho_{e q}$ [22, 23]:

$$
\rho_{e q}=Z_{e q}^{-1} e^{-\beta\left(\mathcal{H}_{0}+H^{\prime}\right)}=\rho_{0} \frac{\widehat{T}_{x} \exp \left[-\int_{0}^{\beta} \widetilde{H}_{I}^{\prime}(x) d x\right]}{\left\langle\widehat{T}_{x} \exp \left[-\int_{0}^{\beta} \widetilde{H}_{I}^{\prime}(x) d x\right]\right\rangle^{0}}=\frac{Z_{0}}{Z_{e q}} \rho_{0} \widetilde{U}(\beta, 0)
$$


where $Z_{e q}=Z_{0}\langle\widetilde{U}(\beta, 0)\rangle^{0}$ and the brackets $\langle\ldots\rangle^{0}=\operatorname{tr}\left[\rho_{0} \ldots\right]$ correspond to the statistical average with respect to $\rho_{0}=Z_{0}^{-1} e^{-\beta \mathcal{H}_{0}}$ with $Z_{0}=\operatorname{tr}\left(e^{-\beta \mathcal{H}_{0}}\right)$.

The following steps depend on the particular values of the arguments $x_{1}$ and $x_{2}$. However, since the Green's function depends only on their difference, $x=x_{1}-x_{2}$, which lies between $-\beta$ and $\beta$, it is sufficient to consider only negative values of $x_{1}$ and $x_{2}$. Thus, combining Eqs. (70) and (72), we can write for the product of the operators in the Green's function (61):

$$
\rho_{e q} \widehat{T}_{x}\left(\bar{A}\left(x_{1}\right) \bar{B}\left(x_{2}\right)\right)=\frac{Z_{0}}{Z_{e q}} \rho_{0} \widetilde{U}(\beta, 0) \widehat{T}_{x}\left[\widetilde{A}_{I}^{\prime}\left(x_{1}\right) \widetilde{B}_{I}^{\prime}\left(x_{2}\right)\right]
$$

The evolution operator $\widetilde{U}(\beta, 0)$ contains the sum of ordered products of operators $\widetilde{H}_{I}^{\prime}(x)$ whose arguments $x$ lie between zero and $\beta$, i.e. are all positive. Since, by our assumption, both $x_{1}$ and $x_{2}$ are negative, the above formula can be transformed into:

$$
\frac{Z_{0}}{Z_{e q}} \rho_{0} \widehat{T}_{x}\left[\widetilde{U}(\beta, 0) \widetilde{A}_{I}^{\prime}\left(x_{1}\right) \widetilde{B}_{I}^{\prime}\left(x_{2}\right)\right]
$$

which results in the following final expression for the Green's function:

$$
G_{A B}\left(x_{1}, x_{2}\right)=-\frac{\left\langle\widehat{T}_{x} \widetilde{U}(\beta, 0) \widetilde{A}_{I}^{\prime}\left(x_{1}\right) \widetilde{B}_{I}^{\prime}\left(x_{2}\right)\right\rangle^{0}}{\langle\widetilde{U}(\beta, 0)\rangle^{0}}=-\frac{\left\langle\widehat{T}_{x} \exp \left[-\int_{0}^{\beta} \widetilde{H}_{I}^{\prime}(x) d x\right] \widetilde{A}_{I}^{\prime}\left(x_{1}\right) \widetilde{B}_{I}^{\prime}\left(x_{2}\right)\right\rangle^{0}}{\left\langle\widehat{T}_{x} \exp \left[-\int_{0}^{\beta} \widetilde{H}_{I}^{\prime}(x) d x\right]\right\rangle^{0}}
$$

which is nothing but the Matsubara Green's function, $\mathcal{G}_{A^{\prime} B^{\prime}}\left(x_{1}, x_{2}\right)$. The latter is defined with respect to the operators $A^{\prime}$ and $B^{\prime}$ which are obtained from the original operators $A$ and $B$ by using the primed matrices of coefficients as explained above.

Thus, there is a direct connection between the Green's function (61) and the appropriate Matsubara Green's function. Since there is a well-known diagrammatic perturbation technique developed for the latter with the denominator cancelling out exactly as a prefactor to connected diagrams in the nominator [22, 23],

$$
G_{A B}\left(x_{1}, x_{2}\right)=-\left\langle\widehat{T}_{x} \exp \left[-\int_{0}^{\beta} \widetilde{H}_{I}^{\prime}(x) d x\right] \widetilde{A}_{I}^{\prime}\left(x_{1}\right) \widetilde{B}_{I}^{\prime}\left(x_{2}\right)\right\rangle_{c}^{0},
$$

where the subscript "c" indicates explicitly that only connected diagrams are to be retained, this method can be directly used to obtain corrections beyond the one-electron approximation. The latter was employed in Section 3.5 to derive the formula for the correlation function. In particular, in the zero order (when the exponential operator above is replaced by unity), the same expression is obtained for the correlation function as in Section 3.5. Notice that the direct application of Eq. (75) results in an expression containing an additional term with the product of averages $\langle A\rangle^{0}\langle B\rangle^{0}$; this term did not appear in Section 3.5 since the correlation funciton considered there contained already the difference operators $\Delta A$ and $\Delta B$.

\section{References}

[1] M. P. Allen and D. J. Tildesley, Computer simulation of liquids (Clarendon Press, Oxford, 2002).

[2] D. C. Rapaport, The art of molecular dynamics simulation (Cambridge Univ. press, Cambridge, 2002).

[3] L. Kantorovich, Quantum theory of the solid state: an introduction.Fundamental Theories of Physics (Kluwer Academic Publishers, Dordrecht, 2004).

[4] R. Car and M. Parrinello, Phys. Rev. Lett. 55, 2471 (1985).

[5] O. V. Prezhdo, J. Chem. Phys. 111, 8366 (1999).

[6] A. P. Horsfield et al., Rep. Prog. Phys. 69, 1195 (2006).

[7] P. Ehrenfest, Z. Phys. 45, 455 (1927).

[8] O. V. Prezhdo, Theor. Chem. Accounts 116, 206 (2006). 
[9] A. P. Horsfield, D. R. Bowler, A. J. Fisher, T. N. Todorov, and C. G. Sánchez, J. Phys. Condens. Matter 16, 8251 (2004).

[10] G. R. Darling and S. Holloway, Rep. Prog. Phys. 58, 1595 (1995).

[11] M. Head-Gordon and J. C. Tully, J. Chem. Phys. 103, 10137 (1995).

[12] D. Zubarev, V. Morozov, and G. Röpke, Statistical mechanics of nonequilibrium processes. Vol. 1: Basic concepts, kinetic theory (Akademie verlag, Berlin, 1996).

[13] L. N. Kantorovich, J. Phys.: Condens. Matter 14, 7123 (2002).

[14] L. N. Kantorovich, Phys. Rev. Lett. 89, 096105 (2002).

[15] I. V. Aleksandrov, Z. Naturforsch A 36A, 902 (1981).

[16] O. V. Prezhdo and V. V. Kisil, Phys. Rev. A 56, 162 (1997).

[17] O. V. Prezhdo and Y. V. Pereverzev, J. Chem. Phys. 113, 6557 (2000).

[18] M. Finnis, P. Agnew, and J. E. Foreman, Phys. Rev. B 44, 567 (1991).

[19] B. Hellsing and M. Persson, Physica Scripta 29, 360 (1984).

[20] A. Liebsch, Phys. Rev. B 55, 13263 (1997).

[21] M. Plihal and D. C. Langreth, Phys. Rev. B 58, 2191 (1998).

[22] A. A. Abrikosov, L. P. Gorkov, and I. E. Dzyaloshinski, Methods of Quantum Field Theory in Statistical Physics (Dover, N. Y., 1975).

[23] D. Zubarev, V. Morozov, and G. Röpke, Statistical mechanics of nonequilibrium processes. Vol. 2: Relaxation and hydrodynamic processes (Akademie verlag, Berlin, 1996).

[24] O. V. Prezhdo, J. Chem. Phys. 124, 201104 (2006). 